\title{
Advances in Systemic Therapy for Metastatic or Advanced Gastric Cancer
}

\author{
Kohei Shitara, MD, and Atsushi Ohtsu, MD, PhD
}

\begin{abstract}
In recent years, various new agents have been investigated for the treatment of advanced gastric cancer (AGC). The anti-HER2 antibody trastuzumab has been shown to prolong the overall survival of patients with HER2-positive AGC and has become a standard treatment. However, lapatinib, or ado-trastuzumab emtansine (T-DM1), did not show survival benefit in AGC, although it has shown remarkable efficacy for HER2-positive breast cancer. The efficacy of the anti-vascular endothelial growth factor receptor monoclonal antibody ramucirumab for pretreated gastric cancer is a milestone for antiangiogenic therapy for AGC. Early clinical trials of TAS-118, TAS-102, and STAT3 inhibitors; IMAB362 (anti-Claudin 18.2); and immune checkpoint inhibitors are all encouraging. These findings warrant further evaluation in larger clinical trials.
\end{abstract}

J Natl Compr Canc Netw 2016;14(10):1313-1320

Systemic chemotherapy is a standard treatment for metastatic or unresectable advanced or recurrent gastric cancer (AGC). Effective cytotoxic agents for AGC include 5-fluorouracil (5-FU), oral fluoropyrimidine, platinum agents, taxanes, irinotecan, and anthracyclines. Commonly used first-line therapy is the combination of fluoropyrimidine and platinum-based chemotherapy with or without docetaxel or anthracycline, which results in median progression-free survival (PFS) of 4 to 6 months and median overall survival (OS) of 10 to 15 months. ${ }^{1-4}$ The survival benefit of second-line therapy has recently been confirmed in several randomized trials. ${ }^{5-7}$ Regarding molecular targeting agents, trastuzum$\mathrm{ab}$, a monoclonal antibody against HER2, significantly improved the OS of HER2-positive AGC, ${ }^{8}$ making this agent standard (Table 1). Ramucirumab, an anti-vascular endothelial growth factor receptor (VEGFR-2) antibody, has proven effective for previously treated AGC. Its addition to best supportive care was associated with significantly improved OS in patients with disease progression on first-line chemotherapy. ${ }^{9}$ Moreover, ramu-

From the Department of Gastrointestinal Oncology, National Cancer Center Hospital East, Kashiwa, Chiba, Japan.

Submitted June 19, 2016; accepted for publication September 26, 2016.

The authors have disclosed that they have no financial interests,

arrangements, affiliations, or commercial interests with the manufacturers of any products discussed in this article or their competitors. cirumab plus paclitaxel chemotherapy significantly increased OS compared with paclitaxel alone in patients after first-line treatment. ${ }^{10}$

Despite the introduction of these new agents, survival in AGC remains unsatisfactory; therefore, the introduction of more effective treatments is desired. Various phase III trials are underway to replace the current standard of care (Table 2). This article reviews the current status of chemotherapy development for AGC.

\section{Molecular Profiles in Gastric Cancer}

Growth factor receptors other than HER2 or receptor tyrosine kinases (RTKs), such as epidermal growth factor receptor (EGFR), mesenchymal-epithelial transition factor (MET; a receptor for hepatocyte growth factor receptor [HGFR]), and fibroblast growth factor receptor (FGFR), have been described. ${ }^{11}$ Detailed molecular profiles of gastric cancer have also been recently reported in large-scale international cancer genome studies. The Cancer Genome Atlas (TCGA) research network used fresh frozen tissues of primary gastric ad-

Correspondence: Kohei Shitara, MD, Department of Gastroenterology and Gastrointestinal Oncology, National Cancer Center Hospital East, 6-5-1,

Kashiwanoha, Kashiwa, Chiba 277-8577, Japan. 
Shitara and Ohtsu

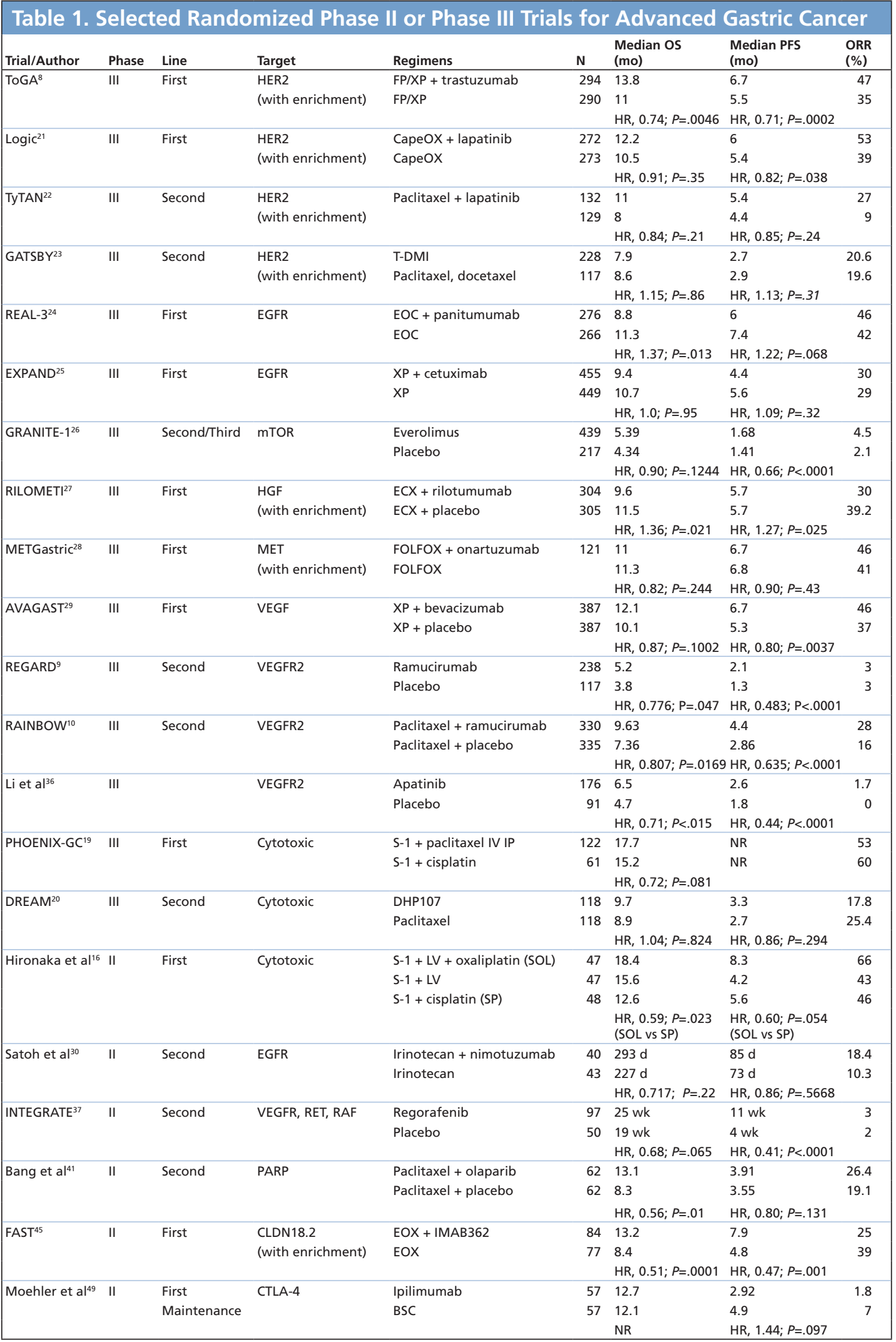

Abbreviations: BSC, best supportive care; CapeOX, capecitabine and oxaliplatin; CTLA-4, cytotoxic T-lymphocyte antigen 4; ECX, epirubicin, cisplatin, and capecitabine; EGFR, epidermal growth factor receptor; EOX, epirubicin, oxaliplatin, and capecitabine; HGFR, hepatocyte growth factor receptor; EOC, epirubicin plus oxaliplatin plus capecitabine; FOLFOX, leucovorin, fluorouracil, and oxaliplatin; FP, 5-FU plus cisplatin; HR, hazard ratio; IP, intraperitoneal; IV, intravenous; LV, leucovorin; MET, mesenchymal-epithelial transition factor; NR, not reported; OS, overall survival; ORR, overall response rate; PARP, poly(ADP-ribose) polymerase; PFS, progression-free survival; T-DM1, trastuzumab emtansine; VEGFR, vascular endothelial growth factor receptor; XP, capecitabine plus cisplatin. 
Systemic Therapy for Gastric Cancer

Table 2. Ongoing Phase III Trials for Advanced Gastric Cancer

\begin{tabular}{|c|c|c|c|c|}
\hline $\begin{array}{l}\text { Trial } \\
\text { (ClinicalTrials.gov Identifier) }\end{array}$ & Line & Control Arm & $\begin{array}{l}\text { Agents } \\
\text { (Experimental) }\end{array}$ & Target \\
\hline $\begin{array}{l}\text { JACOB } \\
\text { (NCT01774786) }\end{array}$ & First & $\mathrm{XP}$ or FP/trastuzumab & + Pertuzumab & HER2 \\
\hline $\begin{array}{l}\text { SOLAR } \\
\text { (NCT02322593) }\end{array}$ & First & S-1 + cisplatin & TAS-118 + oxaliplatin & Cytotoxic \\
\hline $\begin{array}{l}\text { JCOG1013 } \\
\text { (UMIN000007652) }\end{array}$ & First & S-1 + cisplatin & + Docetaxel & Cytotoxic \\
\hline $\begin{array}{l}\text { RAINFALL } \\
\text { (NCT02314117) }\end{array}$ & First & $X P$ & + Ramucirumab & VEGFR2 \\
\hline $\begin{array}{l}\text { KEYNOTE-062 } \\
\text { (NCT02494583) }\end{array}$ & First & $\mathrm{XP} / \mathrm{FP}$ & + Pembrolizumab & PD-1 \\
\hline $\begin{array}{l}\text { JAVELIN Gastric } 100 \\
\text { (NCT02625610) }\end{array}$ & First (maintenance) & Continuation of first line & Avelumab & PD-L1 \\
\hline $\begin{array}{l}\text { ENRICH } \\
\text { (NCT01813253) }\end{array}$ & Second & Irinotecan & + Nimotuzumab & EGFR \\
\hline $\begin{array}{l}\text { GOLD } \\
\text { (NCT01924533) }\end{array}$ & Second & Paclitaxel & + Olaparib & PARP \\
\hline $\begin{array}{l}\text { BRIGHTER } \\
\text { (NCT02178956) }\end{array}$ & Second & Paclitaxel & $+\mathrm{BBI} 608$ & STAT3 \\
\hline $\begin{array}{l}\text { ABSOLUTE } \\
\text { (JapicCTI-132059) }\end{array}$ & Second & Paclitaxel & Nab-paclitaxel & Cytotoxic \\
\hline $\begin{array}{l}\text { KETNOTE-061 } \\
\text { (NCT02370498) }\end{array}$ & Second & Paclitaxel & Pembrolizumab & PD-1 \\
\hline $\begin{array}{l}\text { JAVELIN Gastric } 300 \\
\text { (NCT02625623) }\end{array}$ & Third & Paclitaxel/Irinotecan/BSC & Avelumab & PD-L1 \\
\hline $\begin{array}{l}\text { INTEGRATE-2 (planned) } \\
\text { (NCT02773524) }\end{array}$ & Third or fourth & Placebo & Regorafenib & VEGFR, RET, RAF \\
\hline $\begin{array}{l}\text { ONO-4538-12 } \\
\text { (NCT02267343) }\end{array}$ & Last & Placebo & Nivolumab & PD-1 \\
\hline $\begin{array}{l}\text { Tags } \\
\text { (NCT02500043) }\end{array}$ & Last & Placebo & TAS-102 & Cytotoxic \\
\hline
\end{tabular}

Abbreviations: BSC, best supportive care; EGFR, epidermal growth factor receptor; FP, 5-FU plus cisplatin; PARP, poly(ADP-ribose) polymerase; PD-1, programmed cell death 1; PD-L1, programmed cell death ligand 1; STAT3, signal transducer and activator of transcription-3; VEGFR, vascular endothelial growth factor receptor; XP, capecitabine plus cisplatin.

a Not registered in ClinicalTrials.gov.

enocarcinoma from 295 patients to conduct analyses based on 6 platforms, comprising (1) somatic copynumber alterations, (2) whole-exome sequencing, (3) DNA methylation analysis, (4) mRNA analysis, (5) microRNA analysis, and (6) reverse phase protein array. ${ }^{12}$ Additionally, whole genome analysis and analysis of microsatellite instability (MSI) has been performed. ${ }^{12}$ Four subtypes of gastric cancers have been described: (1) tumors positive for Epstein-Barr virus (EBV), (2) MSI-high (MSI-H) tumors, (3) genomically stable tumors, and (4) tumors with chromosomal instability. The subtype of EBVpositive cancers is characterized by predilection for the gastric corpus, recurrent PIK3CA and ARID1A mutations, high amplification of $9 \mathrm{p}$ chromosomes with increased expression of programmed cell death ligand 1 and 2 (PD-L1/L2), and extreme DNA hypermethylation, whereas the MSI-H subtype shows frequent mutations in multiple genes, such as HER3. The genomically stable subtype has a few somatic copy number alterations involving ARID1A and RHOA mutations or CLDN18-ARHGAP fusions. The subtype with chromosomal instability is rich in TP53 mutations, and has relatively numerous amplifications of RTK genes. Although the TCGA study did not include samples from the Japanese population, a study of 121 Japanese patients with gastric cancer did not demonstrate much difference regarding gene mutations with relatively high frequencies, such as TP53, PIK3CA, ARID1A, CDH1, and gene 
amplifications of RTKs and of those involved in the cell cycle. ${ }^{13}$ From the immunologic viewpoint, Asian gastric cancers showed significantly lower expression of T-cell markers (eg, CD3, CD45RO, CD8), and higher expression of the immunosuppressive transcription factor FOXP3 was reported in Japanese gastric cancers. ${ }^{14}$ In a comprehensive analysis of mostly South Korean patients, a classification of (1) MSI-H, (2) microsatellite stable (MSS)/epithelial mesenchymal transition (EMT), (3) MSS/TP53-positive, and (4) MSS/TP53-negative was also proposed. Particularly in the MSS/EMT subtype, approximately 70\% of recurrences were peritoneal, with a significantly poorer prognosis compared with other subtypes, which highlights the need for therapy development for peritoneal dissemination. ${ }^{15}$

\section{Advances and Ongoing Development in the Treatment for AGC}

\section{Cytotoxic Chemotherapy}

Cytotoxic agents remain important in AGC. A randomized phase II trial of S-1 plus leucovorin (LV) versus $\mathrm{S}-1$ plus $\mathrm{LV}$ and oxaliplatin (SOL) versus S-1 plus cisplatin in patients with AGC showed a higher response rate to SOL with a longer OS (Table 1). ${ }^{16}$ Currently, a phase III SOLAR study comparing TAS-118 (S-1 and LV) plus oxaliplatin with $\mathrm{S}-1$ plus cisplatin is ongoing in Asian countries (ClinicalTrials.gov identifier: NCT02322593). TAS-102 is a novel oral nucleoside antitumor agent containing trifluridine and tipiracil hydrochloride, which prevents the degradation of trifluridine. In a pivotal phase III trial for colorectal cancer, TAS-102 showed an OS benefit compared with placebo in patients with metastatic colorectal cancer that failed to respond to standard chemotherapies. ${ }^{17} \mathrm{~A}$ phase II trial of TAS-102 for pretreated AGC showed a disease control rate of $65.5 \%{ }^{18}$ The median PFS and OS were 2.9 and 8.7 months, respectively. Currently, the ongoing global phase III trial is investigating the efficacy and safety of TAS-102 in patients with AGC refractory to standard treatments (ClinicalTrials.gov identifier: NCT02500043).

The control of peritoneal dissemination is extremely important in gastric cancers, but no breakthrough has yet been obtained. Intraperitoneal paclitaxel (PTX) provides sustained high local concentrations. Based on previous promising results in a phase II trial of intraperitoneal PTX in combination with S-1 plus intravenous PTX, a phase III trial was conducted (PHOENIX-GC). ${ }^{19}$ A total of 183 patients were enrolled, and 164 were included in the efficacy analysis. However, OS was not significantly affected (Table 1). Further study might be necessary to identify suitable candidates for intraperitoneal therapy.

DHP107 is a novel oral lipid formulation of paclitaxel. DREAM is a multicenter, open-label, prospective, randomized phase III study comparing DHP107 and intravenous paclitaxel in AGC after failure of first-line therapy. ${ }^{20}$ Noninferiority of PFS was confirmed. Currently, albumin-bound paclitaxel (nab-paclitaxel) is also being evaluated in a Japanese phase III trial, and results will be presented in the near future.

\section{Molecular Targeting Agents}

Since the emergence of HER2 as a treatment target for AGC, a variety of molecular targeting drugs for EGFR, HGF, MET, and mTOR have been examined. ${ }^{21-30}$ However, most did not demonstrate significant benefit in phase III trials (Table 1). T-DM1, one of the antibody-drug conjugates for HER2 that demonstrates remarkable effectiveness in breast cancer, did not demonstrate an effect on prolonged OS in HER2-positive AGC. ${ }^{23}$ A phase III trial of pertuzum$\mathrm{ab}$, a monoclonal antibody against the dimerization domain of HER2, in combination with cytotoxic chemotherapy and trastuzumab (JACOB), is ongoing (ClinicalTrials.gov identifier: NCT01774786). Although promising results have been obtained in MET inhibitors or FGFR inhibitors for patients with these oncogenic amplifications, ${ }^{31-33}$ these cases are rare. The SCRUM project, ${ }^{34}$ a joint genome screening project in Japan performed in cooperation with major cancer centers and pharmaceutical companies, is underway, and enrollment in clinical studies that deal with patients with rare genomic alterations is anticipated.

Ramucirumab, a fully human immunoglobulin G1 monoclonal antibody against the extracellular VEGF-binding domain of VEGFR-2, has become a standard chemotherapeutic agent for pretreated AGC based on 2 pivotal phase III trials (Table 1), 9,10 although it has not shown success as a first-line agent. ${ }^{35}$ When its efficacy was also evaluated in a randomized phase II study of first-line treatment, it 
Systemic Therapy for Gastric Cancer

did not show an improvement in PFS. ${ }^{35}$ Further, a higher rate of discontinuation from study treatment for reasons other than progressive disease was seen in the ramucirumab arm compared with the placebo arm (50\% vs 19\%), which led to lower study drug exposure in the experimental arm. Currently, the ongoing phase III RAINFALL trial is comparing PFS in patients treated with intensive doses of ramucirumab with cisplatin/capecitabine (or 5-FU) versus placebo with cisplatin/capecitabine (or 5-FU) as first-line treatment (ClinicalTrials.gov identifier: NCT02314117). Apatinib is a multikinase inhibitor that mainly targets VEGFR-2, and which significantly improves $O S$ in patients with pretreated AGC. ${ }^{36}$ Similarly, regorafenib, a multikinase inhibitor, significantly prolonged PFS compared with placebo as second-line or later therapy for AGC. ${ }^{37} \mathrm{~A}$ phase III trial is planned.

Molecular-targeting drugs, such as inhibitors of poly(ADP-ribose) polymerases (PARPs) or of signal transducer and activator of transcription-3 (STAT3) protein, which are factors involved in the resistance mechanism to conventional chemotherapies, are under development for AGC. Clinically, high PARP. 1 expression is associated with tumor invasion and poor prognosis in gastric cancer. ${ }^{38} \mathrm{~A}$ preclinical study using PARP inhibitors showed them to be effective for platinum-resistant cell lines, ${ }^{39}$ and a certain degree of effect has been suggested in ovarian cancer with or without BRCA mutations in clinical studies, ${ }^{40}$ leading to interest in developing agents of this type for patients with AGC. Improved PFS and OS were seen in patients with recurrent or metastatic gastric cancer (Table 1).41 Low expression of ataxia telangiectasia mutated (ATM) protein was suggested to be associated with remarkable efficacy of olaparib. However, the phase III GOLD trial (ClinicalTrials. gov identifier: NCT01924533) that evaluated olaparib in combination with paclitaxel chemotherapy for advanced gastric cancer did not meet its primary end point of improving $O S$ in all cohorts and in an ATM-negative cohort. Detailed reports and biomarker analysis are awaited.

STAT3 is a transcription factor located downstream of a variety of cytokines and JAK, whose expression of STAT3 is associated with a poor prognosis in multiple cancers. ${ }^{42}$ It is reported that phosphorylated STAT3 activates the transcription of Nanog and Myc, genes involved in the "stemness." BBI608 is a drug that specifically inhibits cancer stem-like cells that are highly positive for the glycoprotein CD44 and for STAT3, and that possess the capability to form spheres. ${ }^{42}$ Encouraging anticancer activity of BBI608 and paclitaxel in refractory AGC was observed in a phase Ib and subsequent phase II study including 46 patients with AGC with an overall response rate (ORR) of $31 \%$ and disease control rate of $75 \% .{ }^{43}$ Currently, the phase III BRIGHTER trial (ClinicalTrials.gov identifier: NCT02178956) is ongoing to compare BBI608 plus paclitaxel and placebo plus paclitaxel after first-line chemotherapy for patients with AGC.

Claudin 18.2 (CLDN18.2) is a tight junction protein expressed by several cancers, including gastric adenocarcinoma. IMAB362 is a chimeric monoclonal antibody that mediates specific killing of CLDN18.2-positive cancer cells by activation of immune effector mechanisms. It has shown antitumor activity as monotherapy for pretreated AGC with an ORR of $10 \%$ and response duration of 34 weeks. ${ }^{44} \mathrm{~A}$ randomized phase II trial of IMAB362 in combination with first-line chemotherapy showed promising efficacy for CLDN18.2-positive AGC, ${ }^{45}$ which may warrant evaluation in phase III trials. Efficacy was more prominent in patients with high expression of CLDN18.2.

\section{Immune Checkpoint Inhibitors}

Pembrolizumab is a humanized IgG4 monoclonal antibody without antibody-dependent cytotoxicity (ADCC) activity. It competitively inhibits the binding of PD-1 to PD-L1 and PD-L2. In a phase Ib gastric clinical study (KEYNOTE-012), 39 patients (19 Asians and 20 non-Asians) with PD-L1-positive AGC received pembrolizumab. ${ }^{46}$ Immunohistochemistry with PD-L1 antibody (22C3 antibody) was performed as screening, and those with $1 \%$ or more staining in cancer cells or any staining of stromal cells were assessed as PD-L1-positive. In total, 65 of 162 patients (40\%) were PD-L1-positive. The ORR by central assessment was $22.2 \%$ and that of investigator judgment was $33 \%$, and a reduction in the size of the target lesions was observed in 53.1\% of the patients. Although the median PFS was 1.9 months, the 6 -month survival rate was $69 \%$ and the median response duration was as long as 40 weeks, with an excellent median OS of 11.4 months, despite the fact that $67 \%$ of the patients had received 
2 or more of lines of prior therapy. No significant difference in clinical outcomes was observed between Asians and non-Asians in terms of response, PFS, or OS. Treatment-related adverse events were similar to those of a previous study for another solid tumor. Clinical trials of pembrolizumab are currently underway, including a large phase II trial (KEYNOTE-059; ClinicalTrials.gov identifier: NCT02335411), a phase III trial (KEYNOTE-061; ClinicalTrials.gov identifier: NCT02370498) comparing pembrolizumab with paclitaxel as second-line therapy, and a phase III trial (KEYNOTE-062; ClinicalTrials.gov identifier: NCT02494583) comparing single-agent pembrolizumab with a combination of 5-FU (or capecitabine), cisplatin, and pembrolizumab versus placebo as first-line therapy for patients with PD-L1positive and HER2-negative AGC.

Nivolumab is a humanized $\mathrm{IgG} 4$ recombinant anti-PD-1 monoclonal antibody. In a phase I/II trial (CheckMate-032), 59 patients, 83\% of whom had received 2 or more prior regimens, were treated with nivolumab monotherapy. ${ }^{47}$ The ORR was $14 \%$, median PFS was 1.4 months, and median OS was 5.0 months. The 6-month and 12-month survival rates were $49 \%$ and $36 \%$, respectively. A phase III clinical study of nivolumab in patients with AGC refractory to 2 or more lines of treatment (ONO-4538-12; ClinicalTrials.gov identifier: NCT02267343) has finished its recruitment and the results will be reported in the near future.

In a phase I trial with an expansion cohort of Japanese patients with gastric cancer, the anti-PDL1 antibody avelumab demonstrated an ORR of 15\% with median PFS of 11.9 weeks. ${ }^{48}$ Currently, 2 phase III studies of maintenance therapy after first-line (JAVELIN Gastric 100; ClinicalTrials.gov identifier: NCT02625610) and third-line (JAVELIN Gastric 300; ClinicalTrials.gov identifier: NCT02625623) treatment are ongoing.

Although there have been no established biomarkers of immune checkpoint inhibitors to date, an association has been suggested in several different types of cancers among therapeutic effects and PDL1 expression, types of tumor-infiltrating lymphocytes, number of somatic mutations (mainly passenger mutations), and immune-related gene expression in tumor tissues. At this time, the definitive impact of PD-L1 expression or RNA signature as biomarkers in AGC is not clear ${ }^{46,47}$ and will be analyzed in a larger cohort from ongoing phase III trials.

Because the results mentioned earlier indicate that single-agent response rates in AGC are approximately $10 \%$ to $20 \%$, and that half of patients exhibit early disease progression, it may be more effective to develop combination treatments to improve outcomes. These strategies include combinations of systemic chemotherapy; molecular targeting agents; radiotherapy; immune checkpoint inhibitors, such as cytotoxic T-lymphocyte antigen 4 (CTLA-4), lymphocyte activation gene-3 (LAG-3), and T-cell immunoglobulin domain, mucin domain (Tim-3); inhibitors of suppressive factors, such as indoleamine 2,3-dioxygenase (IDO) or transforming growth factor $\beta$ (TGF- $\beta$ ); and depletors of suppressive lymphocytes, such as chemokine receptor 4 (CCR4) antibody, as well as local injection of oncolytic viruses to enhance the local immune response. Although monotherapy with anti-CTLA-4 ipilimumab did not show superior efficacy as maintenance therapy after first-line chemotherapy compared with best supportive care in a randomized phase II trial (Table 1), ${ }^{49}$ a combination of nivolumab and ipilimumab in the CheckMate-032 trial showed a relatively higher ORR than monotherapy (14\% with nivolumab monotherapy and 26\% with a lower dose of nivolumab in combination with ipilimumab).$^{50}$ Currently, a phase III trial of this combination is in planning.

Combinations of anti-CTLA-4 antibody tremelimumab with anti-PD-L1 antibody MED4736, and single-agent therapies of MED4736 and tremelimumab are also being evaluated for AGC. Regarding the combination of the existing molecular targeting drugs and immune checkpoint inhibitors, a phase I study of ramucirumab combined with pembrolizum$\mathrm{ab}$ is ongoing. ${ }^{51}$ VEGF-A in tumor tissues has been reported to induce the expression of inhibitory molecules and to exhaust CD8-positive T cells in a mouse model, suggesting the possibility of the combined use of immune checkpoint inhibitors and VEGF inhibitors. $^{52}$

\section{Future Perspectives}

Treatment for AGC in the near future depends on the results of ongoing clinical trials. Personalized medicine is expected to become more important, wherein a series of therapies, such as cytotoxic drugs, molecular targeting agents, immunotherapy, and 
intraperitoneal agents are tailored for individual patients according to their tumor profiling and genomic status or immunologic condition. Optimal timing/sequence should also be evaluated in future clinical trials.

\section{References}

1. Van Cutsem E, Moiseyenko VM, Tjulandin S, et al. Phase III study of docetaxel and cisplatin plus fluorouracil compared with cisplatin and fluorouracil as first-line therapy for advanced gastric cancer: a report of the V325 Study Group. J Clin Oncol 2006;24:4991-4997.

2. Cunningham D, Starling N, Rao S, et al. Capecitabine and oxaliplatin for advanced esophagogastric cancer. N Engl J Med 2008;358:36-46.

3. Kang YK, Kang WK, Shin DB, et al. Capecitabine/cisplatin versus 5-fluorouracil/cisplatin as first-line therapy in patients with advanced gastric cancer: a randomised phase III noninferiority trial. Ann Oncol 2009;20:666-673

4. Koizumi W, Narahara H, Hara T, et al. S-1 plus cisplatin versus S-1 alone for first-line treatment of advanced gastric cancer (SPIRITS trial): a phase III trial. Lancet Oncol 2008;9:215-21.

5. Thuss-Patience PC, Kretzschmar A, Bichev D, et al. Survival advantage for irinotecan versus best supportive care as second-line chemotherapy in gastric cancer-a randomised phase III study of the Arbeitsgemeinschaft Internistische Onkologie (AIO). Eur J Cancer 2011;47:2306-2314.

6. Kang JH, Lee SI, Lim do H, et al. Salvage chemotherapy for pretreated gastric cancer: a randomized phase III trial comparing chemotherapy plus best supportive care with best supportive care alone. J Clin Oncol 2012;30:1513-1518.

7. Ford HE, Marshall A, Bridgewater JA, et al. Docetaxel versus active symptom control for refractory oesophagogastric adenocarcinoma (COUGAR-02): an open-label, phase 3 randomised controlled trial. Lancet Oncol 2014;15:78-86.

8. Bang YJ, Van Cutsem E, Feyereislova A, et al. Trastuzumab in combination with chemotherapy versus chemotherapy alone for treatment of HER2 positive advanced gastric or gastro-oesophageal junction cancer (ToGA): a phase 3, open-label, randomised controlled trial. Lancet 2010;376:687697.

9. Fuchs CS, Tomasek J, Yong CJ, et al. Ramucirumab monotherapy for previously treated advanced gastric or gastro-oesophageal junction adenocarcinoma (REGARD): an international, randomised, multicentre, placebo-controlled, phase 3 trial. Lancet 2014; 383:31-39.

10. Wilke H, Muro K, Van Cutsem E, et al. Ramucirumab plus paclitaxel versus placebo plus paclitaxel in patients with previously treated advanced gastric or gastro-oesophageal junction adenocarcinoma (RAINBOW): a doubleblind, randomised phase 3 trial. Lancet Oncol 2014; 15:1224-1235.

11. Nagatsuma AK, Aizawa $M$, Kuwata $T$, et al. Expression profiles of HER2, EGFR, MET and FGFR2 in a large cohort of patients with gastric adenocarcinoma. Gastric Cancer 2015;18:227-238

12. Cancer Genome Atlas Research Network. Comprehensive molecular characterization of gastric adenocarcinoma. Nature 2014;513:202-209.

13. Kuboki $Y$, Yamashita $S$, Niwa $T$, et al. Comprehensive analyses using next-generation sequencing and immunohistochemistry enable precise treatment in advanced gastric cancer. Ann Oncol 2016;27:127-133.

14. Lin SJ, Gagnon-Bartsch JA, Tan IB, et al. Signatures of tumour immunity distinguish Asian and non-Asian gastric adenocarcinomas. Gut 2015;64:1721-1731.

15. Cristescu R, Lee J, Nebozhyn M, et al. Molecular analysis of gastric cancer identifies subtypes associated with distinct clinical outcomes. Nat Med 2015;21:449-456.

16. Hironaka S, Sugimoto N, Yamaguchi K, et al. S-1 plus leucovorin versus S-1 plus leucovorin and oxaliplatin versus S-1 plus cisplatin in patients with advanced gastric cancer: a randomised, multicentre, open-label, phase 2 trial. Lancet Oncol 2016;17:99-108.

17. Mayer RJ, Van Cutsem E, Falcone A, et al. Randomized trial of TAS-102 for refractory metastatic colorectal cancer. N Engl J Med 2015;372:1909_ 1919.

18. Bando H, Doi T, Muro K, et al. A multicenter phase II study of TAS102 monotherapy in patients with pre-treated advanced gastric cancer (EPOC1201). Eur J Cancer 2016;62:46-53.
19. Ishigami H, Fujiwara Y, Fukushima R, et al. Phase III study of intraperitoneal paclitaxel plus s-1/paclitaxel compared with s-1/cisplatin in gastric cancer patients with peritoneal metastasis: PHOENIX-GC trial [abstract]. J Clin Oncol 2016;34(Suppl):Abstract 4014.

20. Kang YK, Ryu MH, Park SH, et al. Efficacy and safety findings from DREAM: a phase III study of DHP107 (oral paclitaxel) vs IV paclitaxel in patients with gastric cancer after failure of first-line chemotherapy [abstract]. J Clin Oncol 2016;34(Suppl):Abstract 4016.

21. Hecht JR, Bang YJ, Qin SK, et al. Lapatinib in combination with capecitabine plus oxaliplatin in human epidermal growth factor receptor 2-positive advanced or metastatic gastric, esophageal, or gastroesophageal adenocarcinoma: TRIO-013/LOGiC—a randomized phase III trial. J Clin Oncol 2016;34:443-451.

22. Satoh T, Xu RH, Chung HC, et al. Lapatinib plus paclitaxel versus paclitaxel alone in the second-line treatment of HER2-amplified advanced gastric cancer in Asian populations: TyTAN-a randomized, phase III study. J Clin Oncol 2014;32:2039-2049.

23. Kang YK, Shah MA, Ohtsu A, et al. A randomized, open-label, multicenter, adaptive phase $2 / 3$ study of trastuzumab emtansine ( $\mathrm{T}$ DM1) versus a taxane (TAX) in patients (pts) with previously treated HER2-positive locally advanced or metastatic gastric/gastroesophageal junction adenocarcinoma (LA/MGC/GEJC) [abstract]. J Clin Oncol 2016;34(Suppl):Abstract 5.

24. Waddell T, Chau I, Cunningham D, et al. Epirubicin, oxaliplatin, and capecitabine with or without panitumumab for patients with previously untreated advanced oesophagogastric cancer (REAL3): a randomised, open-label phase 3 trial. Lancet Oncol 2013;14:481-489.

25. Lordick F, Kang YK, Chung HC, et al. Capecitabine and cisplatin with or without cetuximab for patients with previously untreated advanced gastric cancer (EXPAND): a randomised, open-label phase 3 trial. Lancet Oncol 2013;14:490-499.

26. Ohtsu A, Ajani JA, Bai YX, et al. Everolimus for previously treated advanced gastric cancer: results of the randomized, double-blind, phase III GRANITE-1 study. J Clin Oncol 20131;31:3935-3943.

27. Cunningham D, Tebbutt NC, Davidenko I, et al. Phase III, randomized, double-blind, multicenter, placebo $(\mathrm{P})$-controlled trial of rilotumumab (R) plus epirubicin, cisplatin and capecitabine (ECX) as first-line therapy in patients (pts) with advanced MET-positive (pos) gastric or gastroesophageal junction (G/GEJ) cancer: RILOMET-1 study [abstract]. J Clin Oncol 2015;33(Suppl):Abstract 4000.

28. Shah MA, Bang YJ, Lordick F, et al. METGastric: a phase III study of onartuzumab plus mFOLFOX6 in patients with metastatic HER2negative (HER2-) and MET-positive (MET+) adenocarcinoma of the stomach or gastroesophageal junction (GEC) [abstract]. J Clin Oncol 2015;33(Suppl):Abstract 4012 .

29. Ohtsu A, Shah MA, Van Cutsem E, et al. Bevacizumab in combination with chemotherapy as first-line therapy in advanced gastric cancer: a randomized, double-blind, placebo-controlled phase III study. J Clin Oncol 2011;29:3968-3976.

30. Satoh T, Lee KH, Rha SY, et al. Randomized phase II trial of nimotuzumab plus irinotecan versus irinotecan alone as second-line therapy for patients with advanced gastric cancer. Gastric Cancer 2015;18:824-832.

31. Kwak EL, LoRusso P, Hamid O, et al. Clinical activity of AMG 337, an oral MET kinase inhibitor, in adult patients (pts) with MET-amplified gastroesophageal junction (GEJ), gastric $(\mathrm{G})$, or esophageal (E) cancer [abstract]. J Clin Oncol 2015;33(Suppl 3): Abstract 1.

32. Shitara K, Oh DY, Yokota T, et al. A phase I study of MET TKI SAR125844 in Asian patients (pts) with advanced solid tumors. Proceedings of the AACR-NCI-EORTC International Conference: Molecular Targets and Cancer Therapeutics; November 5-9, 2015; Boston, MA. Philadelphia, PA. AACR; Mol Cancer Ther 2015;14(12 Suppl 2):Abstract A167.

33. Smyth EC, Turner NC, Peckitt C, et al. Phase II multicenter proof of concept study of AZD4547 in FGFR amplified tumours [abstract]. J Clin Oncol 2015;33(Suppl):Abstract 2508.

34. Shitara K, Miki I, Sudo T, et al. The Nationwide Cancer Genome Screening Projects for Gastrointestinal Cancer in Japan (SCRUM-Japan GI-SCREEN): efficient identification of actionable cancer genome alterations in advanced colorectal and non-colorectal gastrointestinal cancer (GI Screen 2013-01-CRC and 2015-01-Non CRC) [abstract]. J Clin Oncol 2015;33(Suppl):Abstract TPS4134.

35. Yoon HH, Bendell JC, Braiteh FC, et al. Ramucirumab (RAM) plus FOLFOX as front-line therapy $(\mathrm{Rx})$ for advanced gastric or esophageal adenocarcinoma (GE-AC): randomized, double-blind, multicenter phase 2 trial [abstract]. J Clin Oncol 201432(Suppl):Abstract 4004. 
36. Li J, Qin S, Xu J, et al. Randomized, double-blind, placebo-controlled phase III trial of apatinib in patients with chemotherapy-refractory advanced or metastatic adenocarcinoma of the stomach or gastroesophageal junction. J Clin Oncol 2016;34:1448-1454.

37. Pavlakis N, Sjoquist KM, Tsobanis E, et al. INTEGRATE: a randomized, phase II, double-blind, placebo-controlled study of regorafenib in refractory advanced oesophagogastric cancer (AOGC): a study by the Australasian Gastrointestinal Trials Group (AGITG)—final overall and subgroup results [abstract]. J Clin Oncol 2015;33(Suppl):Abstract 4003.

38. Liu Y, Zhang Y, Zhao Y, et al. High PARP-1 expression is associated with tumor invasion and poor prognosis in gastric cancer. Available at: https://www.spandidos-publications.com/10.3892/ol.2016.5169. Accessed September 29, 2016.

39. Michels J, Vitale I, Galluzzi L, et al. Cisplatin resistance associated with PARP hyperactivation. Cancer Res 2013;73:2271-2280.

40. Ledermann J, Harter P, Gourley C, et al. Olaparib maintenance therapy in patients with platinum-sensitive relapsed serous ovarian cancer: a preplanned retrospective analysis of outcomes by BRCA status in a randomised phase 2 trial. Lancet Oncol 2014;15:852-861.

41. Bang YJ, Im SA, Lee KW, et al. Randomized, double-blind phase II trial with prospective classification by ATM protein level to evaluate the efficacy and tolerability of olaparib plus paclitaxel in patients with recurrent or metastatic gastric cancer. J Clin Oncol 2015;33:3858-3865.

42. Li Y, Rogoff HA, Keates S, et al. Suppression of cancer relapse and metastasis by inhibiting cancer stemness. Proc Natl Acad Sci U S A 2015;112:1839-1844.

43. Becerra C, Stephenson J, Jonker DJ, et al. Phase Ib/II study of cancer stem cell (CSC) inhibitor BBI608 combined with paclitaxel in advanced gastric and gastroesophageal junction (GEJ) adenocarcinoma [abstract]. J Clin Oncol 2015;33(Suppl):Abstract 4069.

44. Schuler MH, Zvirbule Z, Lordick F, et al. Safety, tolerability, and efficacy of the first-in-class antibody IMAB362 targeting claudin 18.2 in patients with metastatic gastroesophageal adenocarcinomas [abstract]. J Clin Oncol 2013;31(Suppl):Abstract 4080.
45. Al-Batran SE, Schuler MH, Zanete Zvirbule, et al. FAST: an international, multicenter, randomized, phase II trial of epirubicin, oxaliplatin, and capecitabine (EOX) with or without IMAB362, a first-in-class antiCLDN18.2 antibody, as first-line therapy in patients with advanced CLDN18.2+ gastric and gastroesophageal junction (GEJ) adenocarcinoma [abstract]. J Clin Oncol 2016;34(Suppl):Abstract LBA4001.

46. Muro K, Chung HC, Shankaran V, et al. Pembrolizumab for patients with PD-L1-positive advanced gastric cancer (KEYNOTE-012): a multicentre, open-label, phase 1b trial. Lancet Oncol 2016;17:717-726.

47. Le DT, Bendell JC, Calvo E, et al. Safety and activity of nivolumab monotherapy in advanced and metastatic $(\mathrm{A} / \mathrm{M})$ gastric or gastroesophageal junction cancer (GC/GEC): results from the CheckMate-032 study [abstract]. J Clin Oncol 2016;34(Suppl):Abstract 6.

48. Yamada $Y$, Nishina $T$, Iwasa $S$, et al. A phase I dose expansion trial of avelumab (MSB0010718C), an anti-PD-L1 antibody, in Japanese patients with advanced gastric cancer [abstract]. J Clin Oncol 2015;33(Suppl):Abstract 4047.

49. Moehler MH, Cho JY, Kim YH, et al. A randomized, open-label, twoarm phase II trial comparing the efficacy of sequential ipilimumab (ipi) versus best supportive care (BSC) following first-line (1L) chemotherapy in patients with unresectable, locally advanced/metastatic $(\mathrm{A} / \mathrm{M})$ gastric or gastro-esophageal junction (G/GEJ) cancer [abstract]. J Clin Oncol 2016;34(Suppl):Abstract 4011.

50. Janjigian YY, Bendell JC, Calvo E, et al. CheckMate-032: phase I/II, open-label study of safety and activity of nivolumab (nivo) alone or with ipilimumab (ipi) in advanced and metastatic (A/M) gastric cancer (GC) [abstract]. J Clin Oncol 2016;34(Suppl):Abstract 4010.

51. Herbst RS, Bendell JC, Isambert $N$, et al. A phase 1 study of ramucirumab (R) plus pembrolizumab (P) in patients (pts) with advanced gastric or gastroesophageal junction (G/GEJ) adenocarcinoma, non-small cell lung cancer (NSCLC), or urothelial carcinoma (UC): phase 1a results [abstract]. J Clin Oncol 2016;34(Suppl):Abstract 3056.

52. Voron T, Colussi O, Marcheteau E, et al. VEGF-A modulates expression of inhibitory checkpoints on CD8 $+\mathrm{T}$ cells in tumors. J Exp Med 2015;9;212:139-148 